\title{
«Неделя электроники с Siemens": цифровые технологии как конкурентное преимущество
}

\author{
В. Ежов
}

\begin{abstract}
Создание сложной электроники сегодня требует внедрения цифровых методов и технологий на всех этапах жизненного цикла изделия: от идеи и разработки нового продукта до технологической подготовки производства и оперативного планирования производственного процесса. Среди ведущих мировых компаний, успешно внедряющих решения в этой области, только Siemens предлагает полный набор инструментов для автоматизации разработки и производства электроники любой степени сложности. В основе подхода Siemens - концепция интегрированной системы цифровых двойников в цепочке "изделие - технологический процесс - производство - сервисное сопровождение». 25-29 мая 2020 года компания Siemens Digital Industries Software провела серию вебинаров, посвященных обзору цифровых технологий, помогающих сделать разработку и производство электроники максимально эффективными.
\end{abstract}

лектронные продукты становятся все более функционально насыщенными, интеллектуальными и вместе с тем миниатюрными и надежными. В то же время заказчики становятся все более требовательными к качеству новых продуктов и срокам выполнения производственных заказов. В условиях острой конкуренции старые подходы к управлению разработкой и производством электроники перестают работать, не позволяя быстро реагировать на рыночные изменения. Цифровые технологии помогают эффективно справляться с возрастающей сложностью электронных изделий, оптимизировать производственные процессы и тем самым повышать их конкурентоспособность на рынке.

На «Неделе электроники с Siemens» был представлен набор решений компании, охватывающих ключевые направления и этапы разработки и производства электронной аппаратуры любого назначения и сложности. Эксперты Siemens Digital Industries Software рассказали о том, как цифровые технологии позволяют оптимизировать все процессы создания нового продукта и эффективно реализовывать производственные задачи.

Одна из важнейших задач при разработке электроники - интеграция процессов и систем разработки механической и электронной составляющих изделия. Согласно исследованиям компании Aberdeen Group 50\% проектов разработки сложной электроники требуют дополнительной итерации для выявления и решения проблем взаимовлияния электронной и механической частей, а 68\% компаний отмечают, что синхронизация данных электроники, механики и инженерных расчетов - важная задача при разработке электронных изделий и рассматривается, как дополнительная возможность сокращения цикла разработки и ускорения вывода изделия на рынок.

Компании, не способные эффективно решать эту проблему, характеризуются низким уровнем междисциплинарной интеграции от ТЗ до производства, использованием несинхронизированных данных, а также тем, что отрабатывают все изменения в изделии на физических прототипах. Компании, которым удается преодолеть эти ограничения, реализуют более эффективный процесс разработки, основанный на интегрированных цифровых инструментах. Причем этот процесс должен быть скоординирован также в организационном плане, то есть команды разработчиков, технологов, конструкторов должны работать над проектом совместно, синхронизируя данные между собой. Только компания Siemens предлагает подход, который устраняет междисциплинарные барьеры, обеспечивая беспрепятственную интеграцию разработки электроники, механики, программного обеспечения и моделирования в едином процессе, что позволяет сократить цикл проектирования и достичь более высокого качества продукта.

Илья Чайковский, консультант по развитию бизнеca Siemens Digital Industries Software, посвятил свой доклад техническим аспектам взаимодействия механических и электронных САПР при разработке электронных 
изделий. Сегодня наиболее широко распространенными форматами обмена данными между MCAD- и ECAD-системами, в зависимости от сложности проекта и объема передаваемых данных, являются STEP, IDF и IDX. Компания Siemens предлагает четвертый, наиболее продвинутый подход, который обеспечивает передачу еще большего объема данных. Разработанная компанией Siemens многодисциплинарная система проектирования NX поддерживает все четыре формата передачи данных. Интеграция системы NX и инновационной электронной САПР Xpedition от Mentor, A Siemens Business расширяет возможности разработчиков, позволяя проектировать сложные электронные модули с высокой плотностью монтажа. Благодаря поддержке передачи данных о структуре внутренних слоев, закрытых и внутренних отверстиях, атрибутах цепей, радиочастотных и других проводящих элементов на плате достигается наиболее полное и точное представление данных об изделии в обеих системах. Кроме того, интеграция NX и Xpedition расширяет возможности формата IDX, позволяя передавать 3D-модели компонентов между системами. Использование синхронизированной библиотеки компонентов в обеих системaх, NX и Xpedition, дает возможность сократить количество итераций передачи данных и ускорить работу над проектом.

Для разработки механической части сложных электронных изделий нужно применять цифровые технологии, способные реализовать многие ключевые требования и функции, такие как пылевлагозащита, охлаждение, эргономичный дизайн, механическая защита электронной начинки и др. Полнофункциональная система NX обеспечивает комплексный подход к разработке мехатронных систем, сочетая возможности разработки, инженерного анализа и технологической подготовки производства. Илья Чайковский продемонстрировал участникам вебинара важные особенности системы NX, которая обеспечивает совместную работу различных команд над проектом в рамках единой платформы. Система NX полностью интегрирована с PLM-системой Teamcenter, в которой осуществляется управление данными об изделии от этапа разработки до запуска производства. Это обеспечивает актуальность данных, которые формируют цифровой двойник изделия на всех стадиях жизненного цикла изделия.

Среда виртуальной реальности, реализованная в NX, позволяет проводить интерактивный и наглядный анализ 3D-макета изделия. Высокоэффективная среда разработки моделей системы NX предоставляет полный набор инструментов, что обеспечивает гибкость при решении задач любой сложности. В системе поддерживается работа с данными любого формата, благодаря чему значительно сокращается время, поскольку устраняется необходимость в повторном моделировании. Адаптивный пользовательский интерфейс NX обеспечивает 95\%-ную точность предсказания следующих команд, что помогает повысить производительность работы.

Система NX оснащена широким набором средств инженерного анализа, с помощью которых можно выполнять построение сеток, прочностной расчет, анализ гидрогазодинамики и теплопередачи, акустический и кинематический расчет, анализ перемещений и др. Полностью интегрированные среды моделирования и инженерного анализа сокращают число и длительность итераций расчета. Для оптимизации производственных процессов служат инструменты технологической подготовки производства. Например, встроенный в NX инструмент Mold Wizard позволяет оптимизировать конструкцию прессформы и задавать максимально эффективный технологический процесс ее изготовления. Кроме того, с помощью NX можно с успехом решать все задачи аддитивного производства.

Планирование производства - ключевой процесс, влияющий на рентабельность производства. Для учета всех производственных ограничений в планировании нужно принимать во внимание множество факторов: от выбора оборудования и персонала для исполнения заказа до сроков сдачи готовой продукции. Игорь Зарх, консультант по направлению «Оперативное управление

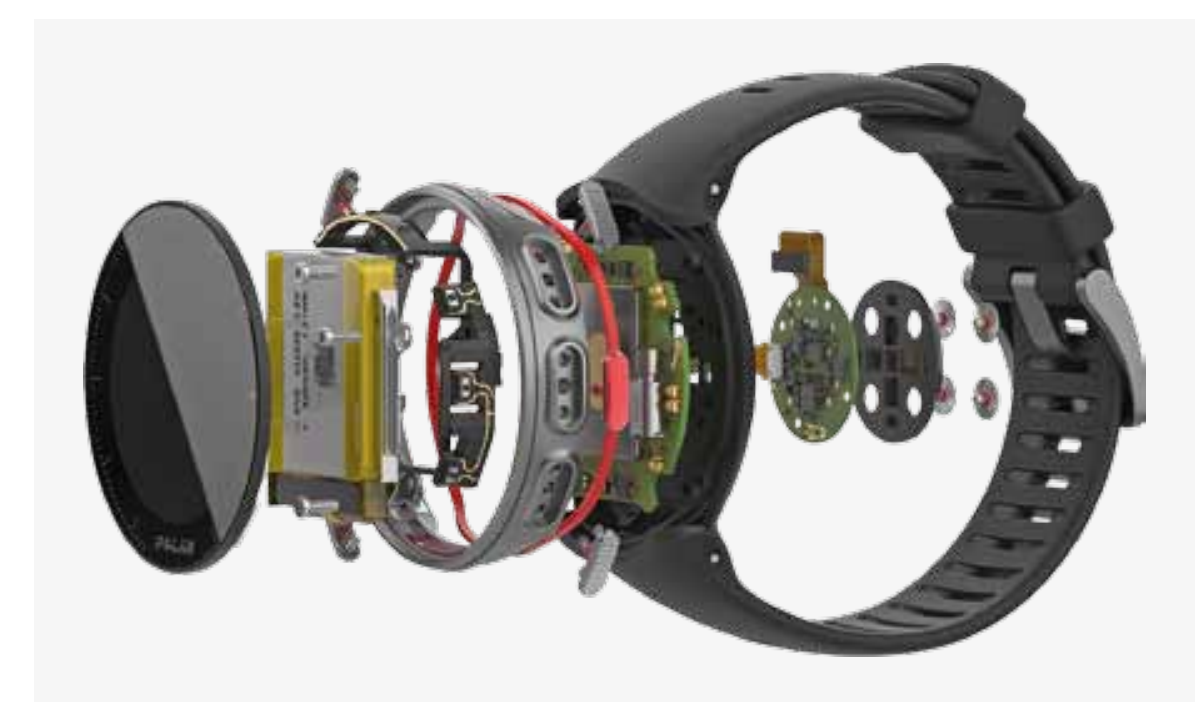

Состоящие из более 500 компонентов часы Polar Vantage спроектированы с помощью интегрированных инструментов Siemens Digital Industries Software 
производством" Siemens Digital Industries Software, представил основные подходы и инструменты для эффективного планирования производства электроники. В состав интегрированного решения от Siemens для приборостроения и электроники входят инструменты для оптимизации производственных расписаний, диспетчеризации и быстрого принятия управляющих решений в условиях динамически меняющегося производственного процесса.

Смоделировать производственный процесс с учетом всех ограничений и выявить незадействованные ресурсы позволяет специальный инструмент Valor Production Plan. Используя подход "что-если», это решение обеспечивает оптимизацию производственного процесса "налету» на основе различных сценариев, например при изменении рабочего календаря, появлении внеплановыхзаказов, добавлении питателей, срыве поставок некоторых компонентов и т.д. Быстро реагировать на какие-либо отклонения в производственном процессе позволяет встроенный в систему «план-факт» анализ. Valor Production Plan эффективно работает совместно с еще одним инструментом - Siemens Opcenter APS (paнеe Preactor), предназначенным для задач по оперативному планированию и диспетчеризации производства. Используя различные методы и алгоритмы планирования, Siemens Opcenter APS предоставляет возможность прогнозировать сроки выполнения заказов, планировать производство с учетом имеющейся загрузки, отслеживать заказы и незавершенное производство на любом этапе, сравнивать варианты расписаний по ключевым параметрам и т.д.

Другая важная задача, требующая решения на современных предприятиях электронной промышленности, управление материалами. С докладом, посвященным методам эффективного управления и улучшения прослеживаемости материалов и изделий по всей цепочке производственного процесса, выступила Регина Субханкулова, консультант по решениям Siemens Digital Industries Software. Первое интегрированное решение для управления материалами от Siemens - Valor Іот Material Management - выполняет весь комплекс задач, связанных с этой проблематикой, включая регистрацию материалов, хранение, комплектацию, проверку. Система, которая взаимодействует с устройствами Интернета вещей, обеспечивает автономное управление материально-технической базой на заводе или фабрике в режиме реального времени. С помощью Valor Іот Material Management возможна передача производственных заказов из ЕRP-системы и отслеживание незавершенного производства в цехе в реальном времени. В результате применения системы достигаются сокращение запасов и отходов, повышение качества материалов, оптимизация потока материалов между складом и цехом, уменьшение времени на перенастройку оборудования, ускоряется расчет потребности в материалах и др.

Регина Субханкулова также познакомила участников вебинара с цифровыми решениями Siemens Digital Industries Software для сбора данных с оборудования и передачи их для анализа состояния предприятия и контроля ключевых показателей производства. Сегодня в электронной промышленности требуются надежные масштабируемые методы сбора больших объемов данных, их хранения и анализа. Возможность сбора и анализа данных в режиме реального времени позволяет цифровому предприятию быстро реагировать на изменения и прогнозировать события, что дает возможность избежать простоев в работе и других проблем с производительностью. Решение Siemens для сбора данных - промышленные компьютеры OpcenterEX EL IоT - обеспечивают связь с разнородным оборудованием, надежный сбор данных (по качеству и выпуску продукции, производительности и загрузке оборудования), приведение данных к единому формату OML, автоматическое управление оборудованием с подключением plug \& play. Благодаря OpcenterEX EL IOT производственные данные в режиме реального времени становятся доступными для корпоративных приложений. На основе собранных данных формируется цифровой двойник производства, обеспечивается полная прослеживаемость материалов и процессов.

Анализ производственных данных предоставляет конкурентные преимущества для компании, поскольку обеспечивает обратную связь по выходу, качеству, затратам, предотвращает возможный возврат продукции от заказчика. Siemens предлагает эффективное решение для производственной аналитики - платформу Valor IoT Manufacturing Analytics, которая помогает находить первопричины проблем и сбоев, позволяет выполнять анализ трендов, предоставляет данные для улучшения цепочек поставок и качества продукции, выбора материала и поставщика и т. д. Результаты, полученные с помощью этого инструмента, дают возможность быстро принимать решения по улучшению всех производственных процессов предприятия.

Siemens предлагает российским компаниям полный набор решений для автоматизации разработки и производства электроники. Внедрение новой платформы не означает для клиента замену всех уже используемых на предприятии систем - заказчик может выбрать нужные ему компоненты и инструменты для решения самых актуальных задач. Российская команда Siemens, обладающая немалым опытом внедрения цифровых технологий, обеспечивает всестороннюю поддержку клиентов. Эффект от внедрения решений Siemens - быстрая окупаемость инвестиций, возможность быстрее конкурентов выводить на рынок качественные продукты, отвечающие высоким требованиям пользователей. 
